\title{
Improving catalyst activity in secondary amine catalysed transformations
}

\author{
John B. Brazier, ${ }^{a}$ Timothy J. K. Gibbs, ${ }^{a}$ Julian H. Rowley, ${ }^{b}$ Leopold Samulis, ${ }^{a}$ \\ Sze Chak Yau, ${ }^{a}$ Alan R. Kennedy, James A. Platts ${ }^{a}$ and \\ Nicholas C. O. Tomkinson ${ }^{b, *}$
}

\begin{abstract}
The effect on catalyst performance of altering substituents at the 2-position of the Macmillan imidazolidinone has been examined. Condensation of L-phenylalanine $N$-methyl amide with acetophenone derivatives results in a series of imidazolidinones whose salts can be used to accelerate the Diels-Alder cycloaddition. Electron withdrawing groups significantly increases the overall rate of cycloaddition without compromise in selectivity. The most effective catalyst was shown to be efficient for a variety of substrates and the applicability of this catalyst to alternative secondary amine catalysed transformations is also discussed.
\end{abstract}

\section{Introduction}

Catalyst design generally relies upon serendipity and extensive high throughput screening which has frequently proved to be effective in asymmetric synthesis. However, pressure to reduce financial and environmental footprints provides inspiration for innovation. The use of predictive models represents the future of asymmetric synthesis whereby bespoke catalysts can be tailored to specific reactions with minimal laboratory screening. Current models, however, frequently fall short of the accuracy required to be effective predictors of both activity and selectivity. ${ }^{1}$ An alternative approach is to draw inspiration from mechanistic knowledge where a combination of kinetic experiment and intelligent design allows interrogation, reinforcing and underpinning of hypotheses. ${ }^{2}$

Amine catalysis represents an important area of contemporary synthetic chemistry. Since their introduction the fields of LUMO, HOMO and SOMO catalysis have been established as the most significant areas of Organocatalysis. ${ }^{3}$ This is due, in part, to the wide variety of reactions which have been developed using secondary amines along with the excellent yields and outstanding levels of selectivity which are commonly achieved in these transformations. ${ }^{4,5}$ The field also follows some of the principles of Green chemistry which has further enhanced applicability, particularly in the industrial environment. Reducing levels of catalyst loading necessary to bring about reaction at an effective rate would further increase this potential. ${ }^{6}$

Within the field of LUMO catalysis using secondary amines a consistent mechanism has been accepted, the fundamental steps of which are outlined in Figure 1.,8,9 In Step 1 the secondary amine salt $\mathbf{1}$ condenses with the $\alpha, \beta$-unsaturated carbonyl compound $\mathbf{2}$ to form the reactive iminium ion $\mathbf{3}$. In Step 2 the diene 4 undergoes cycloaddition with the activated $\pi$-system of $\mathbf{3}$ to form the iminium ion of the product $\mathbf{5}$. Finally, in Step $\mathbf{3}$ the iminium ion $\mathbf{5}$ undergoes hydrolysis/solvolysis to release the product (e.g. acetal 6) and regenerate the secondary amine salt $\mathbf{1}^{10}$

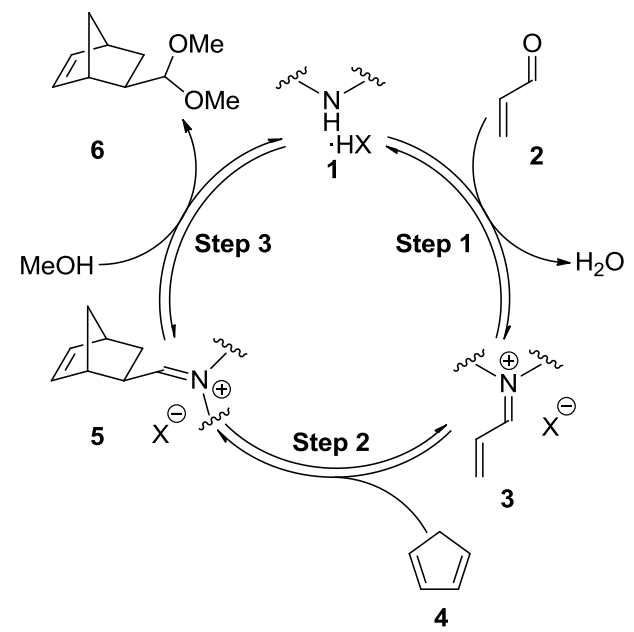

Figure 1. Catalytic cycle for imidazolidinone catalysed DielsAlder cycloaddition.

For the Diels-Alder cycloaddition reaction, under literature optimised conditions, it has been shown the rate determining step of the catalytic cycle is iminium ion formation (Step 1). ${ }^{11}$ Iminium ion formation consists of a number of mechanistic steps, whereby distinct equilibria require the amine to have different properties. ${ }^{12}$ Figure 2 shows a series of productive equilibria from Step 1 leading to a reactive iminium ion 12. Reduced basicity of the amine 7 drives Equilibrium 1 and Equilibrium 4 in the forward direction whereas amine nucleophilicity is required for Equilibrium 3 to proceed forwards. It is well established that the basicity of an amine is intimately linked to its nucleophilicity, therefore, positively influencing Equilibrium 1 and Equilibrium 4 by reducing basicity can negatively impact Equilibrium 3 by reducing 
nucleophilicity. Therefore in order to influence Step 1 of the catalytic cycle the electronics of the amine must be balanced through appropriate substitution.
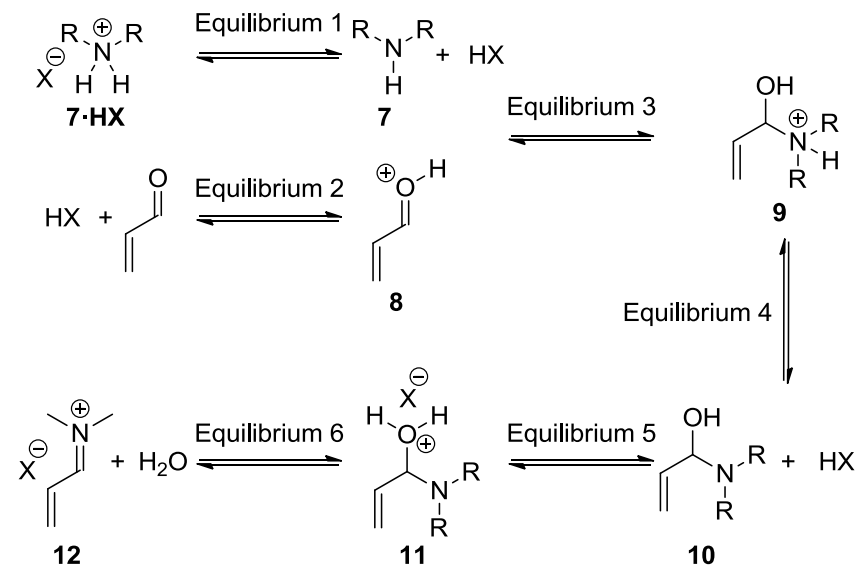

Figure 2. Considerations within Step 1 of the catalytic cycle.

Within the literature there are two principal catalyst scaffolds developed for accelerating the Diels-Alder reaction through iminium ion intermediates (Figure 3): The imidazolidinones $^{13}$ and the diarylprolinol ethers; ${ }^{14}$ the imidazolidinones having a higher level of activity in this reaction. ${ }^{15}$ We therefore elected to examine the imidazolidinone architecture to discover catalysts with higher levels of activity. ${ }^{16,17}$ Within this manuscript we prepare a series of imidazolidinone structures based upon our mechanistic understanding of the reaction and explore the reactivity of these catalysts.

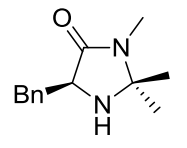

13

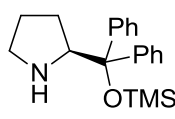

14
PA $943.1 \mathrm{KJ} \mathrm{mol}^{-1}$

PA $998.6 \mathrm{KJ} \mathrm{mol}^{-1}$

Figure 3. Imidazolidinone and diarylprolinol ether catalysts.

Understanding how substituents on the secondary amine can affect basicity, nucleophilicity, and hence the rate of Step 1 was deemed crucial for influencing reactivity. Modelling studies had previously shown the highest activation barrier within iminium ion formation was Equilibrium 1 where the ammonium salt $\mathbf{7} \cdot \mathbf{H X}$ loses a proton (Figure 2). ${ }^{18}$ When comparing the imidazolidinone $\mathbf{1 3}$ and the diarylprolinol ether 14 it was found proton affinity (PA) was a readily calculable theoretical measure of amine basicity to act as a predictor of catalyst activity. ${ }^{15}$ The benchmark imidazolidinone $\mathbf{1 3}$ has a PA of $943.1 \mathrm{KJ} \mathrm{mol}^{-1}$ whereas the diarylprolinol ether 14, which has a lower level activity, has a PA of $998.6 \mathrm{KJ} \mathrm{mol}^{-1}$ (Figure $3) .{ }^{15}$ Therefore we sought to alter the basicity of $\mathbf{1 3}$ through substitution and determine influence on reactivity within a benchmark Diels-Alder reaction, with the expectation that lowering proton affinity would improve reactivity.

The imidazolidinone architecture $\mathbf{1 5}$ offers a number of places for developing an understanding of the relationship between structure and catalytic activity (Figure 4). We elected to examine the substituents $\mathrm{R}^{2}$ and $\mathrm{R}^{3}$ where it was thought ease of synthesis and proximity to the reactive nitrogen would engender the greatest effect on reaction outcome.<smiles>[R]C1NC([R])([R])N([R])C1=O</smiles>

Figure 4. Potential points of substitution in the imidazolidinone architecture.

A second fundamental requirement of the secondary amine catalyst is the ability to control the stereochemical outcome of the transformation. It was crucial for any amine with improved activity over $\mathbf{1 3}$ maintained the exceptional levels of asymmetry generally associated with this catalyst. ${ }^{7}$ Within the reaction of a secondary amine 13 and a $\alpha, \beta$-unsaturated carbonyl compound two potential iminium ions can be formed, the $E$ - and the $Z$ isomers 16 and 17 (Scheme 1). Ratios of 16 and 17 have been shown to be dictated by the steric requirements of the substituents on the imidazolidinone ring. ${ }^{9}$

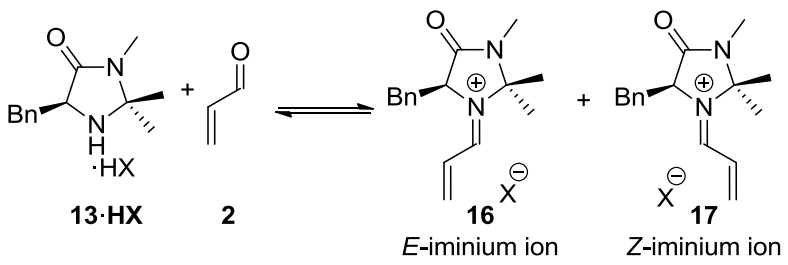

Scheme 1. Origins of stereoselectivity in the imidazolidinone catalysed Diels-Alder cycloaddition.

For the imidazolidinone 13, following selective formation of the $E$ iminium ion 17 , the catalyst architecture renders subsequent Diels-Alder cycloaddition asymmetric, with the benzyl arm of the catalyst directing approach of the diene from the lower face of the iminium ion as shown (Scheme 1). For any alternative imidazolidinone structures it is essential that high levels of $E / Z$ iminium control is observed to maintain levels of selectivity. ${ }^{9 a}$

\section{Results and discussion}

Based upon the hypothesis that introduction of an electron withdrawing group $\alpha$ - to the reactive nitrogen $\left(\mathrm{R}^{2}\right.$ and $\mathrm{R}^{3}$ in 15) would reduce basicity and therefore enhance reactivity a series of imidazolidinones 20-24 were prepared through condensation of phenylalanine- $N$-methyl amide (18) and a substituted acetophenone (Table 1). Reaction of $\mathbf{1 8}$ and $\mathbf{1 9}$ in toluene under microwave irradiation in the presence of ytterbium triflate gave the corresponding imidazolidinones 20 23 with a variety of substitution on the aromatic ring. ${ }^{19}$ For preparation of the imidazolidinone $\mathbf{2 4}$, derived from 4nitroacetophenone, it proved necessary to develop alternative conditions to access the catalyst with high levels of e.e. Reaction of $\mathbf{1 8}$ with the ketone in DMF at $150{ }^{\circ} \mathrm{C}$ for 30 minutes in the presence of methane sulfonic acid gave $\mathbf{2 4}$ enantiomerically pure (Entry 6) (See Supplementary Information for full details of catalyst preparation). Proton affinity (PA) for each catalyst was calculated using Gaussian 09 (B3LYP/6-31+G(d,p)) which showed a clear influence on predicted basicity of the secondary nitrogen (Entries 2-6) when compared to the parent structure 13 (Entry 1). Introduction of an aromatic ring with an electron donating substituent displayed a raised proton affinity (Entry 2,970 $\mathrm{KJ} \mathrm{mol}^{-1}$ ) when compared to 13 (Entry 1, $943 \mathrm{KJ} \mathrm{mol}^{-1}$ ). Whereas increasing the strength 
of electron withdrawing group progressively decreased the proton affinity of the secondary amine (Entries 3-6), with the lowest value being shown for imidazolidinone $24(\mathrm{R}=4$ $\mathrm{NO}_{2} \mathrm{C}_{6} \mathrm{H}_{4}, \mathrm{PA}=924 \mathrm{KJ} \mathrm{mol}^{-1}$ ).

Table 1. Preparation of potential imidazolidinone catalysts. ${ }^{a}$

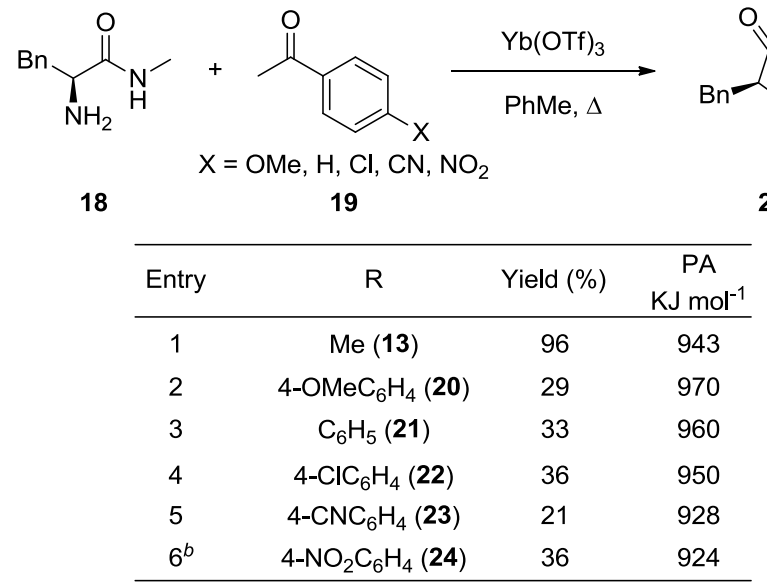

$\mathrm{PA}=$ Proton affinity

${ }^{a}$ Reaction conditions: $\mathrm{Yb}(\mathrm{OTf})_{3}, \mathrm{PhMe}, 120^{\circ} \mathrm{C}, \mu \mathrm{w}$.

${ }^{b}$ Reaction conditions: $\mathrm{MsOH}, \mathrm{DMF}, 150^{\circ} \mathrm{C}, \mu \mathrm{w}, 30 \mathrm{~min}$.

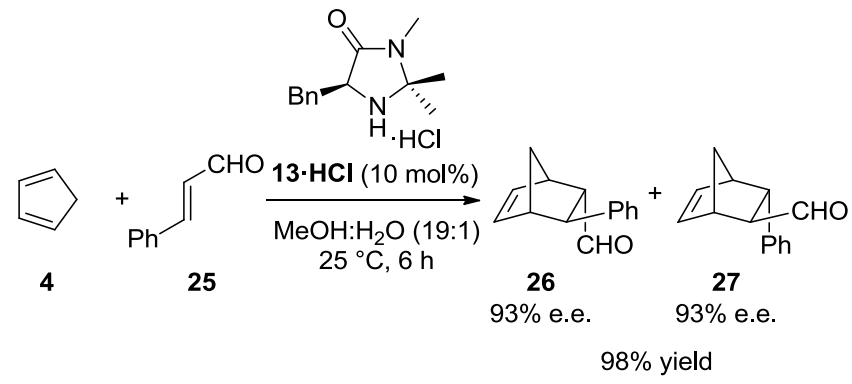

Scheme 2. Diels-Alder cycloaddition catalysed by $\mathbf{1 3} \cdot \mathbf{H C l}$.

The Diels-Alder cycloaddition of cinnamaldehyde and cyclopentadiene was used to benchmark the acetophenone derived catalysts $\mathbf{2 0} \cdot \mathbf{H C l}-\mathbf{2 4} \cdot \mathbf{H C l}$ (Scheme 2$).{ }^{7}$ Each reaction was performed under literature optimised reaction conditions (3 equiv. cyclopentadiene, $\mathrm{MeOH} / \mathrm{H}_{2} \mathrm{O}$ (19:1), $25{ }^{\circ} \mathrm{C}, 5 \mathrm{~mol} \%$ catalyst), monitoring reaction progress by ${ }^{1} \mathrm{H}$ NMR spectroscopy (See Supplementary Information for full details). As can be seen in Figure 5, introduction of an aromatic ring greatly increased the rate of the Diels-Alder cycloaddition when compared to the parent system $\mathbf{1 3} \cdot \mathbf{H C l}(\diamond)$. This was the case for all acetophenone derived catalysts examined. Subtleties in the electronic substitution of the aromatic ring had less influence on the overall rate than proton affinity predictions had suggested (Table 1), showing a deficiency in this ground state prediction, however, the significantly increased reaction rate observed was exciting and warranted further investigation.

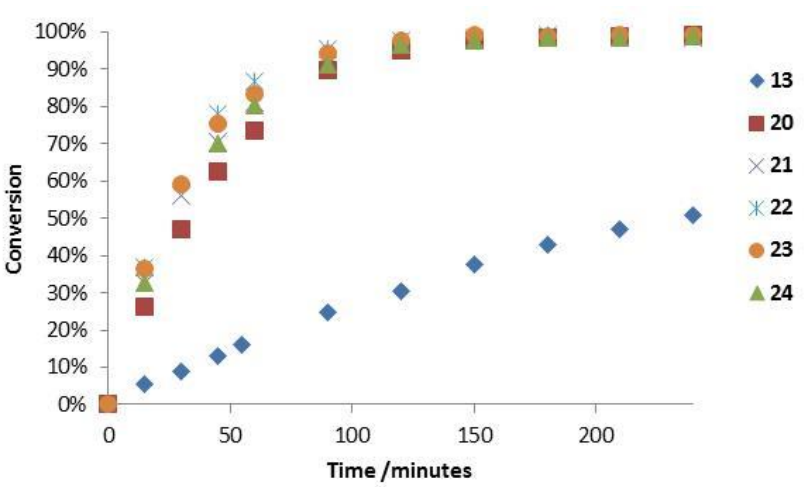

Figure 5. Relative rates of imidazolidinone catalysed DielsAlder cycloaddition.

As stated previously, it was deemed essential that alongside an increase in reaction rate we required the high levels of selectivity observed with the imidazolidinone $\mathbf{1 3} \cdot \mathbf{H C l}$. For each reaction selectivities were determined for the Diels-Alder adducts 26 and 27 (Table 2). ${ }^{20}$ Based upon these reaction outcomes we selected the imidazolidinone derived from 4nitroacetophenone $\mathbf{2 4}$ (Entry 3) for further investigations due to the high levels of enantioselectivity observed for both the endo ( $95 \%$ e.e.) and exo ( $87 \%$ e.e.) isomers of the Diels-Alder product.

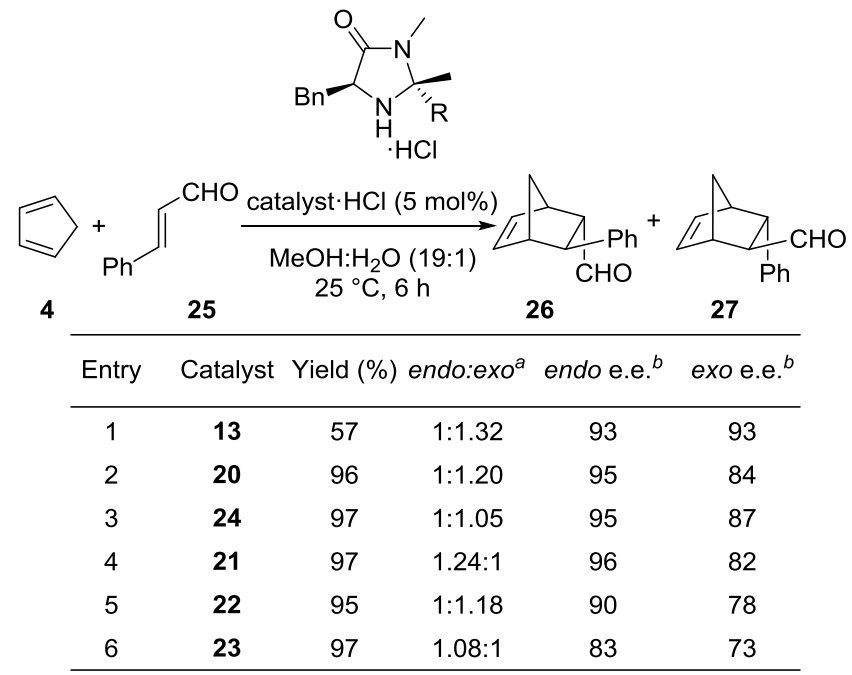

${ }^{a}$ Determined by ${ }^{1} \mathrm{H}$ NMR spectroscopy on crude reaction mixture.

${ }^{b}$ Determined by conversion to 2,4-dinitrophenylhydrazine derivative and examination by HPLC.

Table 2. Imidazolidinone catalysed Diels-Alder cycloaddition.

To profile the reactivity of the imidazolidinone $\mathbf{2 4}$ further we examined a series of alternative dienophiles within the Diels-Alder cycloaddition (Table 3). For cinnamaldehyde derivatives (Entries 1-4) high yields and enantioselectivities were maintained for both electron withdrawing and electron donating substituents on the aromatic ring. Reactions with aliphatic substituted substrates proceeded slower than reactions with cinnamaldehyde derivatives, however, they still progressed cleanly and mass balance could be accounted for through recovered starting material. It is believed that parasitic equilibria of the catalyst involving dienamine $\mathbf{3 0}^{\mathbf{2 1}}$ formation 
from the iminium intermediate $\mathbf{2 9}$ accounts for this observed loss in reactivity (Scheme 3 ).

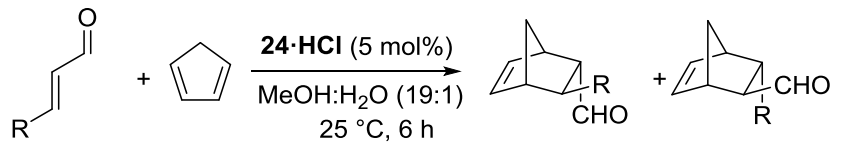

\begin{tabular}{cccccc}
\hline Entry & $\mathrm{R}$ & Yield (\%) & endo:exo & endo e.e. & exo e.e. \\
\hline 1 & $4-\mathrm{OMeC}_{6} \mathrm{H}_{4}$ & 95 & $1: 1.1$ & $96^{c}$ & $87^{c}$ \\
2 & $4-\mathrm{MeC}_{6} \mathrm{H}_{4}$ & 75 & $1: 1$ & $94^{c}$ & $86^{c}$ \\
3 & $4-\mathrm{ClC}_{6} \mathrm{H}_{4}$ & 95 & $1.1: 1$ & $96^{c}$ & $84^{c}$ \\
4 & $4-\mathrm{NO}_{2} \mathrm{C}_{6} \mathrm{H}_{4}$ & 94 & $1.1: 1$ & $94^{c}$ & $86^{c}$ \\
$5^{b}$ & ${ }^{n} \mathrm{Pr}$ & 72 & $1.2: 1$ & $81^{d}$ & $63^{d}$ \\
$6^{b}$ & ${ }^{i} \mathrm{Pr}$ & 18 & $1: 1$ & $80^{d}$ & $64^{d}$ \\
\hline
\end{tabular}

${ }^{a}$ Determined by ${ }^{1} \mathrm{H}$ NMR spectroscopy on crude reaction mixture.

$b_{1} \mathrm{~mol} \% \mathbf{2 4} \cdot \mathbf{H C l}$ used

cDetermined by reduction to corresponding alcohol and examination by HPLC

${ }^{d}$ Determined by conversion to 2,4-dinitrophenylhydrazine derivative and examination by HPLC

Table 3. Diels-Alder cycloaddition catalysed by $\mathbf{2 4} \cdot \mathbf{H C l}$.

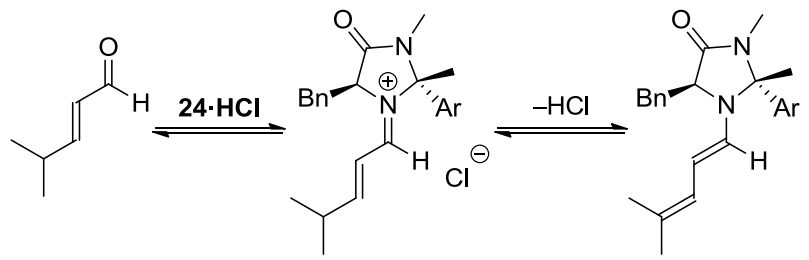

28

29

30

$\mathrm{Ar}=4-\mathrm{NO}_{2} \mathrm{C}_{6} \mathrm{H}_{4}$

Scheme 3. Parasitic dienamine formation.

Crystals of an iminium ion suitable for X-ray analysis were obtained through reaction of $\mathbf{2 4} \cdot \mathrm{HPF}_{6}$ and 4iodocinnamaldehyde (Figure 6). A low energy solid-state conformation of the $E$-iminium ion with the benzyl arm residing over the top of the imidazolidinone ring was observed. Direction of the diene to the bottom face of this iminium ion leads to the stereochemical outcome observed in reactions of 24. ${ }^{22}$ Consistent with observations on the MacMillan catalyst 13, this suggests use of $\mathbf{2 4}$ as a catalyst in conjugate addition reactions would lead to products with low levels of enantioselectivity, ${ }^{23}$ and increasing the steric requirement of the methyl group on the $\beta$-face would be necessary to prepare alternative catalysts to accelerate this class of reaction.

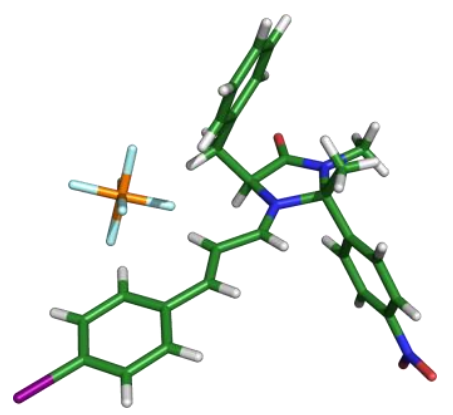

Figure 6. X-Ray structure of iminium ion derived from $\mathbf{2 4}$ and 4-iodocinnamaldehyde.

The importance of knowing the rate determining step of the catalytic cycle when altering catalyst structure is highlighted by examination of an alternative transformation which proceeds via iminium ion intermediates. For example, Mayr has shown that in the conjugate addition of electron rich heteroaromatics, such as $N$-methyl pyrrole $\mathbf{3 1}$, to an $\alpha, \beta$-unsaturated carbonyl compound, $\mathrm{C}-\mathrm{C}$ bond formation is rate determining. ${ }^{24}$ From this it could be expected that the 4-nitroacetophenone derived catalyst $\mathbf{2 4}$ would not significantly alter the rate of addition of 31 to 25 (Scheme 4).

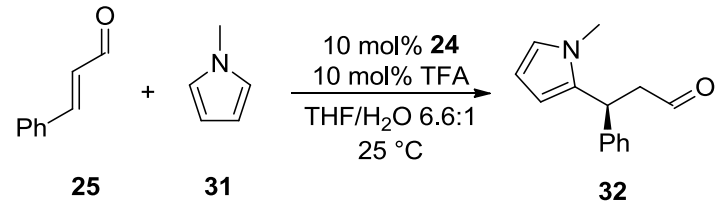

Scheme 4. Conjugate addition of $N$-methyl pyrrole to cinnamaldehyde.

The addition of $\mathbf{3 1}$ to $\mathbf{2 5}$ catalysed by $\mathbf{1 3}$ and $\mathbf{2 4}$ under literature optimised conditions was monitored by ${ }^{1} \mathrm{H}$ NMR spectroscopy (Figure 7). Each reaction proceeded smoothly to completion after 6 hours. As can be seen from Figure 7 each catalyst accelerates the reaction at a similar rate showing that improved activity for $\mathbf{2 4}$ will only be observed in transformations where iminium ion formation is rate determining.

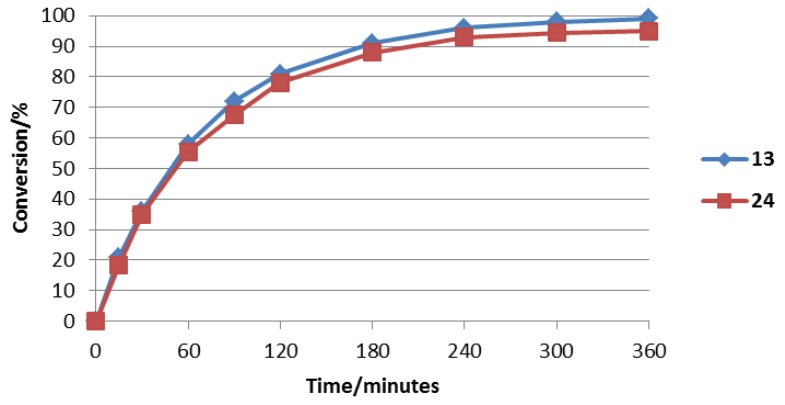

Figure 7. Comparison of the reactivity of $\mathbf{1 3}$ and $\mathbf{2 4}$ in the conjugate addition of $\mathrm{N}$-methyl pyrrole to cinnamaldehyde.

\section{Conclusions}

In summary, we have presented a series of highly reactive imidazolidinone catalysts, which significantly increase the rate of Diels-Alder cycloaddition reactions. A low energy crystal structure of a proposed iminium ion intermediate suggests that salts of $\mathbf{2 4}$ induce asymmetry in a similar manner to the MacMillan imidazolidinone 13. The catalysts described would be predicted to accelerate cycloaddition reactions and conjugate addition reactions that proceed through a closed transition state and provide enhanced rates to those observed with 13 where iminium ion formation is the rate determining step of the catalytic cycle. Central to this method of catalyst design is a thorough understanding of reaction mechanism providing an attractive and practical method to improve catalyst activity. 


\section{Experimental}

L-Phenylalanine $\boldsymbol{N}$-methyl amide 18. L-Phenylalanine ethyl ester hydrochloride $(30.0 \mathrm{~g}, 131 \mathrm{mmol})$ was stirred in an ethanolic solution of methylamine $33 \mathrm{wt} . \%(150 \mathrm{~mL}, 1.2 \mathrm{~mol})$ for 72 hours at ambient temperature. The solvent was removed under reduced pressure. The resultant slurry was taken up in saturated sodium carbonate $(50 \mathrm{~mL})$ and extracted with chloroform $(3 \times 50 \mathrm{~mL})$. The combined organic extracts were dried over anhydrous potassium carbonate and the solvent removed under reduced pressure to give a white solid. The residue was recrystallized from ethyl acetate and petroleum ether to give the product as white needles (17.6 g, 75\%). mp: 67-69 ${ }^{\circ} \mathrm{C} ;[\alpha]_{\mathrm{D}}^{20}=-89.6\left(\mathrm{c}=1, \mathrm{CHCl}_{3}\right) ; \mathrm{v}_{\max }(\mathrm{film}) / \mathrm{cm}^{-1} 3371$, 3345, 3290, 2939, 2875, 1644; ${ }^{1} \mathrm{H}$ NMR (400 MHz, $\left.\mathrm{CDCl}_{3}\right) \delta$ $7.47-7.12(6 \mathrm{H}, \mathrm{m}), 3.54(1 \mathrm{H}, \mathrm{dd}, J=9.5,3.9 \mathrm{~Hz}), 3.22(1 \mathrm{H}, \mathrm{dd}$, $J=13.7,3.9 \mathrm{~Hz}), 2.83(3 \mathrm{H}, \mathrm{d}, J=5.0 \mathrm{~Hz}), 2.60(1 \mathrm{H}, \mathrm{dd}, J=$ 13.7, $9.5 \mathrm{~Hz}), 1.38(2 \mathrm{H}, \mathrm{s}) ;{ }^{13} \mathrm{C} \mathrm{NMR}\left(101 \mathrm{MHz}, \mathrm{CDCl}_{3}\right) \delta$ $174.8,138.0,129.3,128.7,126.8,56.5,41.0,25.8 ; \mathrm{m} / z$ (ES): $179.1\left(\mathrm{M}+\mathrm{H}^{+}\right)$.

\section{(S)-5-Benzyl-2,2,3-trimethylimidazolidin-4-one}

13. L-Phenylalanine- $N$-methyl amide $(2.00 \mathrm{~g}, 11.2 \mathrm{mmol})$ was dissolved in acetone $(10 \mathrm{~mL})$ and methanol $(30 \mathrm{~mL})$. A small crystal of $p$-toluenesulfonic acid $(<1 \mathrm{mg})$ was added and the mixture was heated under reflux for $18 \mathrm{~h}$. The solvent was removed under reduced pressure and the residue taken up in chloroform $(30 \mathrm{~mL})$ and saturated sodium carbonate solution $(30 \mathrm{~mL})$. The aqueous layer was extracted with chloroform $(2 \times$ $30 \mathrm{~mL}$ ) and the combined organics were dried over potassium carbonate. The solvent was removed under reduced pressure to give the target compound as a pale yellow oil (2.40 g, quant.). $[\alpha]_{\mathrm{D}}^{20}=-33.5(\mathrm{c}=1.3, \mathrm{MeOH}) ; v_{\max }($ film $) / \mathrm{cm}^{-1} 3473,3329$, 3060, 3030, 2975, 2929, 1685; ${ }^{1} \mathrm{H}$ NMR (400 MHz, $\left.\mathrm{CDCl}_{3}\right) \delta$ $7.38-7.14(5 \mathrm{H}, \mathrm{m}), 3.82(1 \mathrm{H}, \mathrm{dd}, J=6.5,4.5 \mathrm{~Hz}), 3.17(1 \mathrm{H}, \mathrm{dd}$, $J=14.2,4.5 \mathrm{~Hz}), 3.03(1 \mathrm{H}, \mathrm{dd}, J=14.2,6.5 \mathrm{~Hz}), 2.77(3 \mathrm{H}, \mathrm{s})$, $1.28(3 \mathrm{H}, \mathrm{s}), 1.18(3 \mathrm{H}, \mathrm{s}) ;{ }^{13} \mathrm{C}$ NMR $\left(126 \mathrm{MHz}, \mathrm{CDCl}_{3}\right) \delta$ 173.7, 137.6, 129.9, 129.0, 127.2, 75.9, 59.7, 37.7, 27.6, 25.7, 25.6; $\mathrm{m} / \mathrm{z}(\mathrm{APCI}): 219.2\left(\mathrm{M}+\mathrm{H}^{+}\right)$.

General Procedure for the preparation of imidazolidinones 20-23. A toluene solution of L-Phenylalanine- $N$-methyl amide $(0.6 \mathrm{M})$, the appropriate substituted acetophenone $(0.9$ eq.) and ytterbium(III) trifluoromethanesulfonate $(0.05$ eq.) were heated under reflux for $16-69 \mathrm{~h}$. The mixture was then allowed to cool and diethyl ether ( 2 vol.) was added. The solution was washed with $4 \mathrm{M}$ potassium carbonate $(0.3$ vol. $)$, water $(0.3$ vol. $)$, and brine $(0.3$ vol.). The volatiles were then removed under reduced pressure and the desired products obtained by flash chromatography (ethyl acetate/heptane).

(2R,5S)-5-Benzyl-2-(4-methoxyphenyl)-2,3-

dimethylimidazolidin-4-one 20. Prepared according to General Procedure. $[\alpha]_{\mathrm{D}}^{30}=+36\left(c 0.1, \mathrm{CH}_{2} \mathrm{Cl}_{2}\right) ; v_{\max }(\mathrm{NaCl}$ disk) $/ \mathrm{cm}^{-1}$ 3360, 2933, 1690, 1608, 1510; ${ }^{1} \mathrm{H}$ NMR $(500 \mathrm{MHz}$, $\left.\mathrm{CDCl}_{3}\right) \delta_{\mathrm{H}} 7.23-7.08(7 \mathrm{H}, \mathrm{m}), 6.78(2 \mathrm{H}, \mathrm{d}, J=8.9 \mathrm{~Hz}), 3.81$ $(1 \mathrm{H}, \mathrm{dd}, J=7.2 \mathrm{~Hz}, 4.2 \mathrm{~Hz}), 3.71(3 \mathrm{H}, \mathrm{s}), 3.08(1 \mathrm{H}, \mathrm{dd}, J=13$. $9 \mathrm{~Hz}, 4.2 \mathrm{~Hz}), 2.93(1 \mathrm{H}, \mathrm{dd}, J=13.9 \mathrm{~Hz}, 7.2 \mathrm{~Hz}), 2.64(3 \mathrm{H}, \mathrm{s})$, $1.46(3 \mathrm{H}, \mathrm{s}) ;{ }^{13} \mathrm{C}$ NMR $\left(125 \mathrm{MHz}, \mathrm{CDCl}_{3}\right) \delta_{\mathrm{C}} 173.6,159.4$, $137.7,134.9,129.7,128.5,126.7,126.5,114.1,78.4,59.4$, 55.3, 38.4, 26.4, 26.1; LRMS $m / z(E S)(\mathrm{M}+\mathrm{H})^{+}=311.2$; HRMS $\mathrm{m} / \mathrm{z}(\mathrm{ES})\left(\mathrm{M}+\mathrm{H}^{+}\right)=311.1753$; HRMS $\mathrm{m} / \mathrm{z}$ calc. $\left(\mathrm{M}+\mathrm{H}^{+}\right)=$ 311.1760
(2R,5S)-5-Benzyl-2,3-dimethyl-2-phenylimidazolidin-4-one 21. Prepared according to General Procedure. $[\alpha]_{\mathrm{D}}^{30}=+3.0(c$ $\left.0.1, \mathrm{CH}_{2} \mathrm{Cl}_{2}\right) ; v_{\max }(\mathrm{NaCl}$ disk $) / \mathrm{cm}^{-1} 1693,1454 ;{ }^{1} \mathrm{H} \mathrm{NMR}(500$ $\left.\mathrm{MHz}, \mathrm{CDCl}_{3}\right) \delta_{\mathrm{H}} 7.23(2 \mathrm{H}, \mathrm{t}, J=6.2 \mathrm{~Hz}), 7.20-7.09(8 \mathrm{H}, \mathrm{m})$, $3.76(1 \mathrm{H}, \mathrm{dd}, J=7.1 \mathrm{~Hz}, 4.2 \mathrm{~Hz}), 3.05(1 \mathrm{H}, \mathrm{dd}, J=13.9 \mathrm{~Hz}$, $4.2 \mathrm{~Hz}), 2.91(1 \mathrm{H}, \mathrm{dd}, J=13.9 \mathrm{~Hz}, 7.1 \mathrm{~Hz}), 2.63(3 \mathrm{H}, \mathrm{s}), 1.45$ $(3 \mathrm{H}, \mathrm{s}) ;{ }^{13} \mathrm{C}$ NMR $\left(125 \mathrm{MHz}, \mathrm{CDCl}_{3}\right) \delta_{\mathrm{C}} 173.7,142.8,137.6$, $129.7,128.9,128.5,128.1,126.7,125.1,78.6,59.3,38.3,26.3$, 26.2; LRMS $\mathrm{m} / \mathrm{z}$ (ES) $(\mathrm{M}+\mathrm{H})^{+}=281.2$; HRMS $\mathrm{m} / \mathrm{z}$ (ES) $\left(\mathrm{M}+\mathrm{H}^{+}\right)=281.1646 ;$ HRMS $m / z$ calc. $\left(\mathrm{M}+\mathrm{H}^{+}\right)=281.1654$.

\section{R,5S)-5-Benzyl-2-(4-chlorophenyl)-2,3-}

dimethylimidazolidin-4-one 22. Prepared according to General Procedure. $[\alpha]_{\mathrm{D}}^{30}=+5.2\left(c 0.1, \mathrm{CH}_{3} \mathrm{OH}\right) ; v_{\max }(\mathrm{NaCl}$ disk) $/ \mathrm{cm}^{-1} 3316,2720,1704,1493 ;{ }^{1} \mathrm{H}$ NMR $(500 \mathrm{MHz}$, $\left.\mathrm{CDCl}_{3}\right) \delta_{\mathrm{H}} 7.23-7.19(4 \mathrm{H}, \mathrm{m}), 7.16-7.14(3 \mathrm{H}, \mathrm{m}), 7.13-7.09$ $(2 \mathrm{H}, \mathrm{m}), 3.76(1 \mathrm{H}, \mathrm{dd}, J=6.9 \mathrm{~Hz}, 4.3 \mathrm{~Hz}), 3.06(1 \mathrm{H}, \mathrm{dd}, J=$ $14.0 \mathrm{~Hz}, 4.3 \mathrm{~Hz}), 2.95(1 \mathrm{H}, \mathrm{dd}, J=14.0 \mathrm{~Hz}, 6.9 \mathrm{~Hz}), 2.64(3 \mathrm{H}$, s), $1.44(3 \mathrm{H}, \mathrm{s}) ;{ }^{13} \mathrm{C} \mathrm{NMR}\left(125 \mathrm{MHz}, \mathrm{CDCl}_{3}\right) \delta_{\mathrm{C}} 173.5,141.4$, $137.3,134.1,129.7,129.0,128.6,126.8,126.7,78.2,59.2$, 38.0, 26.2, 21.0; LRMS $m / z$ (ES) $\left(\mathrm{M}+\mathrm{H}^{+}\right)=315.1$; HRMS $\mathrm{m} / \mathrm{z}$ (ES) $\left(\mathrm{M}+\mathrm{H}^{+}\right)=315.1253$; HRMS m/z calc. $\left(\mathrm{M}+\mathrm{H}^{+}\right)=$ 315.1264 .

\section{$(2 R, 5 S)-5-B e n z y l-2,3-d i m e t h y l-2-(4-$}

cyanophenyl)imidazolidin-4-one 23. Prepared according to General Procedure. $[\alpha]_{\mathrm{D}}^{20}=+41.8\left(c 0.1, \mathrm{CH}_{3} \mathrm{OH}\right) ; v_{\max }(\mathrm{NaCl}$ disk) $/ \mathrm{cm}^{-1}$ 2228, 1695; ${ }^{1} \mathrm{H}$ NMR $\left(500 \mathrm{MHz}, \mathrm{CDCl}_{3}\right) \delta_{\mathrm{H}} 7.57$ $(2 \mathrm{H}, \mathrm{dt}, J=10.4 \mathrm{~Hz}, 1.7 \mathrm{~Hz}), 7.31(2 \mathrm{H}, \mathrm{dt}, J=10.4 \mathrm{~Hz}, 1.7$ $\mathrm{Hz}), 7.24(2 \mathrm{H}, \mathrm{t}, J=7.5 \mathrm{~Hz}), 7.20-7.15(3 \mathrm{H}, \mathrm{m}), 3.72(1 \mathrm{H}, \mathrm{dd}$, $J=6.3 \mathrm{~Hz}, 4.7 \mathrm{~Hz}), 3.07(1 \mathrm{H}, \mathrm{dd}, J=14.1 \mathrm{~Hz}, 4.7 \mathrm{~Hz}), 3.00$ $(1 \mathrm{H}, \mathrm{dd}, J=14.1 \mathrm{~Hz}, 6.3 \mathrm{~Hz}), 2.99(3 \mathrm{H}, \mathrm{s}), 1.94(1 \mathrm{H}, \mathrm{s}), 1.45$ $(3 \mathrm{H}, \mathrm{s}) ;{ }^{13} \mathrm{C}$ NMR $\left(125 \mathrm{MHz}, \mathrm{CDCl}_{3}\right) \delta_{\mathrm{C}} 173.6,147.9,136.8$, $132.7,129.6,128.7,127.0,126.1,118.3,112.1,78.2$, 59.0, 37.6, 26.3, 25.8; LRMS $\mathrm{m} / z$ (APCI) $\left(\mathrm{M}+\mathrm{H}^{+}\right)=306.2$; HRMS $\mathrm{m} / \mathrm{z}$ (ES) $\left(\mathrm{M}+\mathrm{H}^{+}\right)=306.1600 ;$ HRMS $m / z$ calc. $\left(\mathrm{M}+\mathrm{H}^{+}\right)=$ 306.1606 .

\section{(2R,5S)-5-Benzyl-2,3-dimethyl-2-(4-}

nitrophenyl)imidazolidin-4-one 24. L-Phenylalanine- $N$-methyl amide (3.86 g, $21.7 \mathrm{mmol})$ was dissolved in DMF (20 mL). 4Nitroacetophenone $(3.96 \mathrm{~g}, 24 \mathrm{mmol}$ ) was added followed by methanesulfonic acid $(0.3 \mathrm{~mL}, 4.63 \mathrm{mmol}, 20 \mathrm{~mol} \%)$. The mixture was separated into two microwave vials and subjected to microwave irradiation at $150{ }^{\circ} \mathrm{C}$ for 30 minutes. The contents of the two vials were combined and concentrated under reduced pressure. Chloroform $(40 \mathrm{~mL})$ and saturated sodium carbonate solution $(40 \mathrm{~mL})$ were added. The aqueous layer was extracted with chloroform $(2 \times 40 \mathrm{~mL})$ and dried over anhydrous potassium carbonate. The solvent was removed under reduced pressure and the residue purified by flash chromatography (20\% ethyl acetate in petroleum ether) to give the title compound as an orange oil $(500 \mathrm{mg}, 7 \%) .[\alpha]_{\mathrm{D}}^{20}=+24.2(\mathrm{c}=2.0$, $\mathrm{MeOH}) ; v_{\max }(\mathrm{ATR}) / \mathrm{cm}^{-1} 3350,3028,2924,2855,1688 ;{ }^{1} \mathrm{H}$ NMR (400 MHz, $\left.\mathrm{CDCl}_{3}\right) \delta 8.26-8.16(2 \mathrm{H}, \mathrm{m}), 7.51-7.44(2 \mathrm{H}$, $\mathrm{m}), 7.36-7.22(5 \mathrm{H}, \mathrm{m}), 3.83(1 \mathrm{H}$, app. $\mathrm{t}, J=5.3 \mathrm{~Hz}), 3.18(1 \mathrm{H}$, dd, $J=14.1,4.6 \mathrm{~Hz}), 3.11(1 \mathrm{H}, \mathrm{dd}, J=14.1,6.5 \mathrm{~Hz}), 2.80(3 \mathrm{H}$, s), $2.04(1 \mathrm{H}, \mathrm{s}), 1.57(3 \mathrm{H}, \mathrm{s}) ;{ }^{13} \mathrm{C} \mathrm{NMR}\left(101 \mathrm{MHz}, \mathrm{CDCl}_{3}\right) \delta$ $173.7,150.0,147.8,136.9$, 129.8, 128.8, 127.1, 126.5, 124.2, 78.2, 59.1, 37.7, 26.4, 26.1; $\mathrm{m} / \mathrm{z}$ (ES/APCI): $326.2\left(\mathrm{M}+\mathrm{H}^{+}\right)$.

General Procedure for imidazolidinone salt formation. A solution of the amine in diethyl ether was treated with hydrogen chloride gas for 10 minutes. The precipitate was recovered on a 
sinter, washed with diethyl ether and petroleum ether, then dried under reduced pressure.

\section{(S)-5-Benzyl-2,2,3-trimethylimidazolidin-4-one}

hydrochloride $13 \cdot \mathbf{H C l}$. mp: $104-106{ }^{\circ} \mathrm{C} ;[\alpha]_{\mathrm{D}}^{20}=-71.5(\mathrm{c}=1.3$, $\mathrm{MeOH}) ; v_{\max }(\mathrm{ATR}) / \mathrm{cm}^{-1} 3416,3380,1710 ;{ }^{1} \mathrm{H}$ NMR (400 $\mathrm{MHz}, \mathrm{MeOD}) \delta$ 7.49-7.27 (5H, m), 4.67 (1H, dd, $J=10.7,3.5$ $\mathrm{Hz}), 3.53(1 \mathrm{H}, \mathrm{dd}, J=15.2,3.5 \mathrm{~Hz}), 3.09(1 \mathrm{H}, \mathrm{dd}, J=15.2$, $10.7 \mathrm{~Hz}), 2.92(3 \mathrm{H}, \mathrm{s}), 1.75(3 \mathrm{H}, \mathrm{s}), 1.60(3 \mathrm{H}, \mathrm{s}) ;{ }^{13} \mathrm{C} \mathrm{NMR}$ (101 MHz, MeOD) $\delta 170.4,139.2,132.9,132.7,131.3,81.7$,

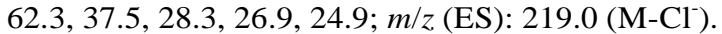

\section{(2R,5S)-5-Benzyl-2-(4-methoxyphenyl)-2,3-}

dimethylimidazolidin-4-one hydrochloride $20 \cdot \mathbf{H C l}$. mp 117 $119^{\circ} \mathrm{C} ;[\alpha]_{\mathrm{D}}^{30}=+36.0\left(c 0.1, \mathrm{CH}_{3} \mathrm{OH}\right) ; v_{\max }(\mathrm{NaCl}$ disk $) / \mathrm{cm}^{-1}$ 3418, 1715, 1608; ${ }^{13} \mathrm{C}$ NMR $\left(125 \mathrm{MHz}, \mathrm{CD}_{3} \mathrm{OD}\right) \delta_{\mathrm{C}} 165.8$, $160.1,133.4,127.3,127.1,126.3,125.8,123.6,113.1,78.5$,

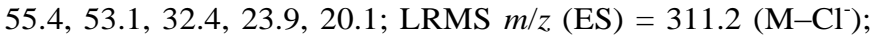
HRMS $m / z$ (ES) $\left(\mathrm{M}-\mathrm{Cl}^{-}\right)=311.1753$; HRMS $m / z$ calc. $\left(\mathrm{M}-\mathrm{Cl}^{-}\right)$ $=311.1760$.

(2R,5S)-5-Benzyl-2,3-dimethyl-2-phenylimidazolidin-4-one hydrochloride $21 \cdot \mathbf{H C l}$. mp $139-141{ }^{\circ} \mathrm{C}$; $[\alpha]_{\mathrm{D}}^{30}=+7.6(c \quad 0.1$, $\left.\mathrm{CH}_{2} \mathrm{Cl}_{2}\right) ; v_{\max }(\mathrm{NaCl}$ disk $) / \mathrm{cm}^{-1} 3412,2096,1644 ;{ }^{1} \mathrm{H} \mathrm{NMR}$ $\left(500 \mathrm{MHz}, \mathrm{CD}_{3} \mathrm{OD}\right) \delta_{\mathrm{H}} 7.60-7.55(3 \mathrm{H}, \mathrm{m}), 7.50-7.46(2 \mathrm{H}, \mathrm{m})$, $7.41(2 \mathrm{H}, \mathrm{d}, J=7.6 \mathrm{~Hz}), 7.36(2 \mathrm{H}, \mathrm{t}, J=7.6 \mathrm{~Hz}), 7.30(1 \mathrm{H}, \mathrm{tt}, J$ $=7.60 \mathrm{~Hz}, 2.4 \mathrm{~Hz}), 4.44(1 \mathrm{H}, \mathrm{dd}, J=9.7 \mathrm{~Hz}, 4.0 \mathrm{~Hz}), 3.57(1 \mathrm{H}$, $\mathrm{dd}, J=15.2 \mathrm{~Hz}, 4.0 \mathrm{~Hz}), 3.24(1 \mathrm{H}, \mathrm{dd}, J=15.2 \mathrm{~Hz}, 9.7 \mathrm{~Hz})$, $2.94(3 \mathrm{H}, \mathrm{s}), 2.21(3 \mathrm{H}, \mathrm{s}) ;{ }^{13} \mathrm{C} \mathrm{NMR}\left(125 \mathrm{MHz}, \mathrm{CD}_{3} \mathrm{OD}\right) \delta_{\mathrm{C}}$ $167.6,134.9,134.3,130.8,129.5,129.0,128.8,127.4,126.9$, 80.5, 58.6, 34.0, 26.2, 19.4; LRMS $\mathrm{m} / \mathrm{z}(\mathrm{ES})=281.2\left(\mathrm{M}-\mathrm{Cl}^{-}\right)$; HRMS $m / z(E S)\left(\mathrm{M}-\mathrm{Cl}^{-}\right)=281.1646$; HRMS $m / z$ calc. $\left(\mathrm{M}-\mathrm{Cl}^{-}\right)$ $=281.1654$.

(2R,5S)-5-Benzyl-2-(4-chlorophenyl)-2,3dimethylimidazolidin-4-one hydrochloride $22 \cdot \mathbf{H C l}$. mp 133 $135{ }^{\circ} \mathrm{C} ;[\alpha]_{\mathrm{D}}^{30}=+38.0\left(c 0.1, \mathrm{CH}_{3} \mathrm{OH}\right) ; v_{\max }(\mathrm{NaCl}$ disk $) / \mathrm{cm}^{-1}$ 3414, 1720, 1495; ${ }^{1} \mathrm{H}$ NMR (500 MHz, CD $\left.{ }_{3} \mathrm{OD}\right) \delta_{\mathrm{H}} 7.58(2 \mathrm{H}$, $\mathrm{dt}, J=8.8 \mathrm{~Hz}, 1.9 \mathrm{~Hz}), 7.47(2 \mathrm{H}, \mathrm{dt}, J=8.8 \mathrm{~Hz}, 1.9 \mathrm{~Hz}), 7.42$ $(2 \mathrm{H}, \mathrm{d}, J=7.4 \mathrm{~Hz}), 7.36(2 \mathrm{H}, \mathrm{t}, J=7.4 \mathrm{~Hz}), 7.30(1 \mathrm{H}, \mathrm{t}, J=7.4$ $\mathrm{Hz}), 4.46(1 \mathrm{H}, \mathrm{dd}, J=9.7 \mathrm{~Hz}, 4.0 \mathrm{~Hz}), 3.57(1 \mathrm{H}, \mathrm{dd}, J=15.2$ $\mathrm{Hz}, 4.0 \mathrm{~Hz}), 3.25(1 \mathrm{H}, \mathrm{dd}, J=15.24 \mathrm{~Hz}, 9.7 \mathrm{~Hz}), 2.93(3 \mathrm{H}, \mathrm{s})$, $2.20(3 \mathrm{H}, \mathrm{s}) ;{ }^{13} \mathrm{C} \mathrm{NMR}\left(125 \mathrm{MHz}, \mathrm{CD}_{3} \mathrm{OD}\right) \delta_{\mathrm{C}} 167.3,136.8$, $134.9,132.4,129.6,129.0,128.7,128.2,127.3,79.6,58.0$, $33.8,25.5,22.2$; LRMS $m / z($ APCI $)=315.1\left(\mathrm{M}-\mathrm{Cl}^{-}\right)$; HRMS $m / z(\mathrm{ES})\left(\mathrm{M}-\mathrm{Cl}^{-}\right)=315.1257$; HRMS m/z calc. $\left(\mathrm{M}-\mathrm{Cl}^{-}\right)=$ 315.1264 .

(2R,5S)-5-Benzyl-2,3-dimethyl-2-(4cyanophenyl)imidazolidin-4-one hydrochloride $23 \cdot \mathbf{H C l}$. mp $109-111{ }^{\circ} \mathrm{C} ;[\alpha]_{\mathrm{D}}^{26}=+43.6\left(c \quad 0.1, \mathrm{CH}_{3} \mathrm{OH}\right) ; v_{\max }(\mathrm{NaCl}$ disk $) / \mathrm{cm}^{-1}$ 3415, 2534, 2231, 1722; ${ }^{1} \mathrm{H}$ NMR $(500 \mathrm{MHz}$, $\left.\mathrm{CD}_{3} \mathrm{OD}\right) \delta_{\mathrm{H}} 7.91(2 \mathrm{H}, \mathrm{d}, J=8.6 \mathrm{~Hz}), 7.65(2 \mathrm{H}, \mathrm{d}, J=8.6 \mathrm{~Hz})$, $7.40(2 \mathrm{H}, \mathrm{d}, J=7.5 \mathrm{~Hz}), 7.34(2 \mathrm{H}, \mathrm{t}, J=7.5 \mathrm{~Hz}), 7.28(1 \mathrm{H}, \mathrm{t}, J$ $=7.5 \mathrm{~Hz}), 4.44(1 \mathrm{H}, \mathrm{dd}, J=9.6 \mathrm{~Hz}, 3.9 \mathrm{~Hz}), 3.55(1 \mathrm{H}, \mathrm{dd}, J=$ $15.2 \mathrm{~Hz}, 3.9 \mathrm{~Hz}), 3.23(1 \mathrm{H}, \mathrm{dd}, J=15.2 \mathrm{~Hz}, 9.6 \mathrm{~Hz}), 2.93(3 \mathrm{H}$, s), $2.21(3 \mathrm{H}, \mathrm{s}) ;{ }^{13} \mathrm{C}$ NMR $\left(125 \mathrm{MHz}, \mathrm{CD}_{3} \mathrm{OD}\right) \delta_{\mathrm{C}} 167.4,138.7$, $134.9,133.2,129.0,128.7,127.7,127.3,117.3,114.5,79.3$, 58.1, 33.8, 25.6, 22.1; LRMS $\mathrm{m} / z($ APCI $)=306.1\left(\mathrm{M}-\mathrm{Cl}^{-}\right)$; HRMS $m / z$ (ES) $\left(\mathrm{M}-\mathrm{Cl}^{-}\right)=306.1597$; HRMS $m / z$ calc. $\left(\mathrm{M}-\mathrm{Cl}^{-}\right)$ $=306.1606$;
2R,5S)-5-Benzyl-2,3-dimethyl-2-(4nitrophenyl)imidazolidin-4-one hydrochloride $\mathbf{2 4} \cdot \mathbf{H C l}$. mp $153-155{ }^{\circ} \mathrm{C} ;[\alpha]_{\mathrm{D}}^{20}=+36.4(\mathrm{c}=2.0, \mathrm{MeOH}) ; v_{\max }(\mathrm{ATR}) / \mathrm{cm}^{-1}$ 1732; $\left.{ }^{1} \mathrm{H} \mathrm{NMR} \mathrm{(400} \mathrm{MHz,} \mathrm{CDCl}_{3}\right) \delta 8.26(2 \mathrm{H}, \mathrm{d}, J=8.8 \mathrm{~Hz})$, $7.58(2 \mathrm{H}, \mathrm{d}, J=8.8 \mathrm{~Hz}), 7.44-7.27(5 \mathrm{H}, \mathrm{m}), 4.28$ (1H, app. t, $J$ $=5.7 \mathrm{~Hz}), 3.43(1 \mathrm{H}, \mathrm{dd}, J=15.1,5.7 \mathrm{~Hz}), 3.31(1 \mathrm{H}, \mathrm{dd}, J=$ 15.1, 5.7 Hz), $2.80(3 \mathrm{H}, \mathrm{s}), 1.82(3 \mathrm{H}, \mathrm{s}) ;{ }^{13} \mathrm{C}$ NMR $(101 \mathrm{MHz}$, $\left.\mathrm{CDCl}_{3}\right) \delta 167.5,149.3,140.9,134.3,130.1,129.1,128.3$, 128.1, 124.6, 79.3, 58.0, 34.6, 26.6, 23.3; m/z (ES/APCI): 326.1 $\left(\mathrm{M}-\mathrm{Cl}^{-}\right.$). HRMS (ES) calculated for $\mathrm{C}_{18} \mathrm{H}_{20} \mathrm{O}_{3} \mathrm{~N}_{3} 326.1499$ $\left(\mathrm{M}+\mathrm{H}^{+}\right)$, found 326.1498 .

General Procedure for Diels Alder cycloaddition (Tables 2 and 3). Imidazolidinone hydrochloride salt (0.1 mmol, $5 \mathrm{~mol} \%)$ was dissolved in a 19:1 methanol/water mixture $(2 \mathrm{~mL})$. The flask was placed into a $25^{\circ} \mathrm{C}$ oil bath. The appropriate aldehyde $(2.0 \mathrm{mmol})$ was added and the mixture was stirred for 10 minutes before freshly distilled cyclopentadiene $(420 \mu \mathrm{L}, 5.0$ mmol) was added in one portion. The reaction mixture was stirred for $6 \mathrm{~h}$ and then the volatiles were removed under reduced pressure. The residue was taken up into chloroform (10 $\mathrm{mL})$ and water $(10 \mathrm{~mL})$ and the aqueous layer was extracted with chloroform $(2 \times 10 \mathrm{~mL})$. The combined organics were dried over sodium sulfate and the solvent was removed under reduced pressure. Chloroform $(2 \mathrm{~mL})$, water $(1 \mathrm{~mL})$ and TFA $(1 \mathrm{~mL})$ were added and the biphasic mixture was vigorously stirred for 2 hours. Potassium carbonate $(20 \mathrm{~mL})$ was added and the mixture was extracted with chloroform $(3 \times 10 \mathrm{~mL})$. The combined organics were dried over sodium sulfate and the solvent was removed under reduced pressure. The products were isolated using flash chromatography (10\% ethyl acetate in petroleum ether) as oils.

\section{Monitoring Diels-Alder cycloaddition between} cinnamaldehyde and cyclopentadiene (Figure 5). Secondary amine salt $(0.25 \mathrm{mmol}, 5 \mathrm{~mol} \%)$ was dissolved in methanol $(4.75 \mathrm{~mL})$ and water $(0.25 \mathrm{~mL})$. The mixture was stirred at 25 ${ }^{\circ} \mathrm{C}$. After 5 minutes, cinnamaldehyde $(630 \mu \mathrm{L}, 5 \mathrm{mmol})$ was added. After another 10 minutes, freshly distilled cyclopentadiene $(1020 \mu \mathrm{L}, 12.5 \mathrm{mmol})$ was added and the reaction timer started. $100 \mu \mathrm{L}$ aliquots were periodically removed and concentrated under reduced pressure $\left(25{ }^{\circ} \mathrm{C}, 15\right.$ torr, 10 minutes). Water $(5 \mathrm{~mL})$ was added and extracted with diethyl ether $(3 \times 5 \mathrm{~mL})$. The combined organics were concentrated under reduced pressure. Chloroform $(2 \mathrm{~mL})$ was added followed by a mixture of TFA/water 1:1 $(2 \mathrm{~mL})$. The biphasic mixture was vigorously stirred for $2 \mathrm{~h}$. The reaction was quenched with saturated sodium carbonate solution $(5 \mathrm{~mL})$ and extracted with diethyl ether $(3 \times 5 \mathrm{~mL})$. The combined organics were dried over magnesium sulfate and the solvent was removed under reduced pressure to give a yellow oil. ${ }^{1} \mathrm{H}$ NMR $\left(\mathrm{CDCl}_{3}\right)$ was used to assess reaction conversion from the CHO resonances: exo $9.93 \mathrm{ppm}(1 \mathrm{H}, \mathrm{d}, J=2.0 \mathrm{~Hz}, \mathrm{CHO})$, cinnamaldehyde $9.71 \mathrm{ppm}(1 \mathrm{H}, \mathrm{d}, J=7.7 \mathrm{~Hz}, \mathrm{CHO})$ and endo $9.60 \mathrm{ppm}(1 \mathrm{H}, \mathrm{d}, J=2.2 \mathrm{~Hz}, \mathrm{CHO})$. The Diels-Alder adducts can be isolated by flash chromatography $(20 \%$ ethyl acetate in petroleum ether) to give a pale yellow viscous oil.

endo-3-Phenylbicyclo[2.2.1] hept-5-ene-2-carbaldehyde 26 and exo-3-phenylbicyclo[2.2.1] hept-5-ene-2-carbaldehyde 27. endo/exo mixture $v_{\max }($ film $) / \mathrm{cm}^{-1} 3414,2972,1717$; exo ${ }^{1} \mathrm{H}$ NMR (500 MHz, $\left.\mathrm{CDCl}_{3}\right) \delta 9.93(1 \mathrm{H}, \mathrm{d}, J=2.0 \mathrm{~Hz}), 7.41-7.12$ $(5 \mathrm{H}, \mathrm{m}), 6.35(1 \mathrm{H}, \mathrm{dd}, J=5.5,3.5 \mathrm{~Hz}), 6.09(1 \mathrm{H}, \mathrm{dd}, J=5.5$, $2.9 \mathrm{~Hz}), 3.77-3.72(1 \mathrm{H}, \mathrm{m}), 3.26-3.21(2 \mathrm{H}, \mathrm{m}), 2.62-2.58(1 \mathrm{H}$, 
$\mathrm{m}), 1.66-1.55(2 \mathrm{H}, \mathrm{m})$; endo ${ }^{1} \mathrm{H}$ NMR $\left(500 \mathrm{MHz}, \mathrm{CDCl}_{3}\right) \delta$ $9.61(1 \mathrm{H}, \mathrm{d}, J=2.2 \mathrm{~Hz}), 7.38-7.11(5 \mathrm{H}, \mathrm{m}), 6.43(1 \mathrm{H}, \mathrm{dd}, J=$ 5.6, 3.2 Hz), $6.19(1 \mathrm{H}, \mathrm{dd}, J=5.6,2.8 \mathrm{~Hz}), 3.35(1 \mathrm{H}$, br. s), 3.16-3.09 $(1 \mathrm{H}, \mathrm{m}), 3.03-2.96(1 \mathrm{H}, \mathrm{m}), 1.60-1.55(2 \mathrm{H}, \mathrm{m})$; endo/exo mixture ${ }^{13} \mathrm{C}$ NMR $\left(126 \mathrm{MHz}, \mathrm{CDCl}_{3}\right) \delta 203.4,202.8$, $143.6,142.7,139.3,136.6,136.4,133.9,128.7,128.2,127.9$, 127.4, 126.4, 126.3, 60.9, 59.5, 48.5, 48.4, 47.6, 47.2, 45.8, 45.5, 45.5, 45.2; $\mathrm{m} / \mathrm{z}(\mathrm{EI}): 198.1\left(\mathrm{M}^{+}\right)$.

\section{endo-3-(4-Methoxyphenyl)bicyclo[2.2.1]hept-5-ene-2- carbaldehyde and exo-3-(4- methoxyphenyl)bicyclo[2.2.1] hept-5-ene-2-carbaldehyde \\ Table 3 Entry 1. endo/exo mixture $v_{\max }(\mathrm{film}) / \mathrm{cm}^{-1}: 2965$, 2934, 2907, 2833, 1712; exo ${ }^{1} \mathrm{H}$ NMR (500 $\left.\mathrm{MHz}, \mathrm{CDCl}_{3}\right) \delta$ $9.92(1 \mathrm{H}, \mathrm{d}, J=2.1 \mathrm{~Hz}, \mathrm{CHO}), 7.10-7.06(2 \mathrm{H}, \mathrm{m}), 6.83-6.79$ $(2 \mathrm{H}, \mathrm{m}), 6.35(1 \mathrm{H}, \mathrm{dd}, J=5.6,3.2 \mathrm{~Hz}), 6.08(1 \mathrm{H}, \mathrm{dd}, J=5.6$, $2.9 \mathrm{~Hz}), 3.78(3 \mathrm{H}, \mathrm{s}), 3.67(1 \mathrm{H}, \mathrm{dd}, J=5.1,3.7 \mathrm{~Hz}), 3.22(1 \mathrm{H}$, $\mathrm{s}), 3.18(1 \mathrm{H}, \mathrm{s}), 2.56-2.53(1 \mathrm{H}, \mathrm{m}), 1.64-1.60(1 \mathrm{H}, \mathrm{m}), 1.58-$ $1.54(1 \mathrm{H}, \mathrm{m})$; endo ${ }^{1} \mathrm{H}$ NMR $\left(500 \mathrm{MHz}, \mathrm{CDCl}_{3}\right) \delta 9.59(1 \mathrm{H}, \mathrm{d}$, $J=2.3 \mathrm{~Hz}, \mathrm{CHO}), 7.22-7.18(2 \mathrm{H}, \mathrm{m}), 6.88-6.84(2 \mathrm{H}, \mathrm{m}), 6.42$ $(1 \mathrm{H}, \mathrm{dd}, J=5.6,3.2 \mathrm{~Hz}), 6.17(1 \mathrm{H}, \mathrm{dd}, J=5.6,2.8 \mathrm{~Hz}), 3.80$ $(3 \mathrm{H}, \mathrm{s}), 3.33(1 \mathrm{H}, \mathrm{s}), 3.08(1 \mathrm{H}, \mathrm{s}), 3.04(1 \mathrm{H}, \mathrm{d}, J=4.5 \mathrm{~Hz})$, 2.96-2.93 $(1 \mathrm{H}, \mathrm{m}), 1.80(1 \mathrm{H}$, app. d, $J=8.7 \mathrm{~Hz}), 1.64-1.60$ $(1 \mathrm{H}, \mathrm{m})$; endolexo mixture ${ }^{13} \mathrm{C}$ NMR $\left(126 \mathrm{MHz}, \mathrm{CDCl}_{3}\right) \delta$ 203.8, 203.0, 158.3, 158.1, 139.4, 136.7, 136.4, 135.7, 134.8, $133.8,128.9,128.4,114.1,113.7,61.0,59.8,55.4,55.4,48.8$, $48.7,47.7,47.2,45.6,45.2,45.2,44.9$; due to fragmentation, mass spectrometric analysis of the parent aldehydes was not possible; $m / z$ of corresponding alcohols $(\mathrm{CI}): 231.1\left(\mathrm{M}+\mathrm{H}^{+}\right)$.}

endo-3-(4-Methylphenyl)bicyclo[2.2.1]hept-5-ene-2carbaldehyde and exo-3-(4methylphenyl)bicyclo[2.2.1]hept-5-ene-2-carbaldehyde

Table 3 Entry 2. endo/exo mixture $v_{\max }(\mathrm{film}) / \mathrm{cm}^{-1}$ : 2968, 2941, 2918, 2874, 2912, 2710, 1715; exo: ${ }^{1} \mathrm{H}$ NMR (500 MHz, $\left.\mathrm{CDCl}_{3}\right) \delta 9.92(1 \mathrm{H}, \mathrm{d}, J=2.1 \mathrm{~Hz}, \mathrm{CHO}), 7.09-7.03(4 \mathrm{H}, \mathrm{m})$, $6.34(1 \mathrm{H}, \mathrm{dd}, J=5.7,3.2 \mathrm{~Hz}), 6.08(1 \mathrm{H}, \mathrm{dd}, J=5.7,2.9 \mathrm{~Hz})$, $3.69(1 \mathrm{H}, \mathrm{dd}, J=5.2,3.2 \mathrm{~Hz}), 3.24-3.19(2 \mathrm{H}, \mathrm{m}), 2.57(1 \mathrm{H}$, app. dt, $J=5.2,1.8 \mathrm{~Hz}), 2.31(3 \mathrm{H}, \mathrm{s}), 1.64-1.53(2 \mathrm{H}, \mathrm{m})$; endo: ${ }^{1} \mathrm{H}$ NMR $\left(500 \mathrm{MHz}, \mathrm{CDCl}_{3}\right) \delta 9.60(1 \mathrm{H}, \mathrm{d}, J=2.3 \mathrm{~Hz}), 7.20$ $7.11(4 \mathrm{H}, \mathrm{m}), 6.42(1 \mathrm{H}, \mathrm{dd}, J=5.6,3.2 \mathrm{~Hz}), 6.17(1 \mathrm{H}, \mathrm{dd}, J=$ 5.6, $2.8 \mathrm{~Hz}), 3.33(1 \mathrm{H}, \mathrm{s}), 3.10(1 \mathrm{H}, \mathrm{s}), 3.05(1 \mathrm{H}, \mathrm{d}, J=4.6 \mathrm{~Hz})$, $2.98-2.95(1 \mathrm{H}, \mathrm{m}), 2.33(3 \mathrm{H}, \mathrm{s}), 1.81(1 \mathrm{H}$, app. d, $J=8.7 \mathrm{~Hz})$, $1.64-1.53(1 \mathrm{H}, \mathrm{m})$; endo/exo mixture: ${ }^{13} \mathrm{C}$ NMR $(126 \mathrm{MHz}$, $\left.\mathrm{CDCl}_{3}\right) \delta 203.7,203.0,140.6,139.7,139.3,136.7,136.4$, $136.0,135.9,133.9,129.4,129.0,127.9,127.4,60.9,59.6$, $48.7,48.5,47.7,47.2,45.6,45.5,45.2,21.0$; due to fragmentation, mass spectrometric analysis of the parent aldehydes was not possible; $\mathrm{m} / \mathrm{z}$ of corresponding alcohol (CI): $215.1\left(\mathrm{M}+\mathrm{H}^{+}\right)$.

\section{endo-3-(4-Chlorophenyl)bicyclo[2.2.1]hept-5-ene-2-

carbaldehyde and exo-3-(4-
Chlorophenyl)bicyclo[2.2.1]hept-5-ene-2-carbaldehyde Table 3 Entry 3. endo/exo mixture $v_{\max }(\mathrm{film}) / \mathrm{cm}^{-1}: 3061$, 2970, 2872, 2810, 2712, 1715; exo: ${ }^{1} \mathrm{H}$ NMR $(500 \mathrm{MHz}$, $\left.\mathrm{CDCl}_{3}\right) \delta 9.91(1 \mathrm{H}, \mathrm{d}, J=1.8 \mathrm{~Hz}, \mathrm{CHO}), 7.24-7.20(2 \mathrm{H}, \mathrm{m})$, $7.10-7.06(2 \mathrm{H}, \mathrm{m}), 6.36(1 \mathrm{H}, \mathrm{dd}, J=5.6,3.2 \mathrm{~Hz}), 6.06(1 \mathrm{H}, \mathrm{dd}$, $J=5.6,2.9 \mathrm{~Hz}), 3.71(1 \mathrm{H}, \mathrm{dd}, J=5.1,3.6 \mathrm{~Hz}), 3.26-3.22(1 \mathrm{H}$, $\mathrm{m}), 3.19$ (1H, br. s), 2.52-2.55 $(1 \mathrm{H}, \mathrm{m}), 1.62-1.56(2 \mathrm{H}, \mathrm{m})$; endo: ${ }^{1} \mathrm{H} \mathrm{NMR}\left(500 \mathrm{MHz}, \mathrm{CDCl}_{3}\right) \delta 9.60(1 \mathrm{H}, \mathrm{d}, J=2.1 \mathrm{~Hz})$, $7.30-7.26(2 \mathrm{H}, \mathrm{m}), 7.22-7.19(2 \mathrm{H}, \mathrm{m}), 6.42(1 \mathrm{H}, \mathrm{dd}, J=5.7$, $3.2 \mathrm{~Hz}), 6.18(1 \mathrm{H}, \mathrm{dd}, J=5.7,2.8 \mathrm{~Hz}), 3.36(1 \mathrm{H}, \mathrm{s}), 3.12-3.08$
$(1 \mathrm{H}, \mathrm{m}), 3.07(1 \mathrm{H}, \mathrm{d}, J=4.1 \mathrm{~Hz}), 2.95-2.90(1 \mathrm{H}, \mathrm{m}), 1.77(1 \mathrm{H}$, app. d, $J=8.7 \mathrm{~Hz}), 1.67-1.63(1 \mathrm{H}, \mathrm{m})$; endo/exo mixture: ${ }^{13} \mathrm{C}$ NMR $\left(126 \mathrm{MHz}, \mathrm{CDCl}_{3}\right) \delta 203.1,202.4,142.2,141.2,139.3$, $136.6,136.4,133.9,132.2,132.1,129.3,128.8,128.8,128.3$, $61.1,59.7,48.5,48.4,47.7,47.2,45.6,45.2,45.2,44.9 ; \mathrm{m} / \mathrm{z}$ (EI): $232.0\left(\mathrm{M}^{+}\right)$; HRMS (EI) calculated for $\mathrm{C}_{14} \mathrm{H}_{13} \mathrm{OCl}^{35}$ 232.0649 $\left(\mathrm{M}^{+}\right)$, found 232.0652.

endo-3-(4-Nitrophenyl)bicyclo[2.2.1]hept-5-ene-2carbaldehyde and exo-3-(4-nitrophenyl)bicyclo[2.2.1]hept5-ene-2-carbaldehyde Table 3 Entry 4. endo/exo mixture $v_{\max }$ (film) $/ \mathrm{cm}^{-1}$ : 3063, 2972, 2943, 2872, 2818, 2717, 1713, 1514, 1342; exo: ${ }^{1} \mathrm{H}$ NMR (500 MHz, $\left.\mathrm{CDCl}_{3}\right) \delta 9.90(1 \mathrm{H}, \mathrm{d}, J=1.4$ $\mathrm{Hz}, \mathrm{CHO}), 8.07(2 \mathrm{H}, \mathrm{d}, J=8.7 \mathrm{~Hz}), 7.28(2 \mathrm{H}, \mathrm{d}, J=8.7 \mathrm{~Hz})$, $6.39(1 \mathrm{H}, \mathrm{dd}, J=5.6,3.2 \mathrm{~Hz}), 6.03(1 \mathrm{H}, \mathrm{dd}, J=5.6,2.8 \mathrm{~Hz})$, 3.88-3.84 (1H, m), $3.28(1 \mathrm{H}, \mathrm{s}), 3.24(1 \mathrm{H}, \mathrm{s}), 2.61$ (1H, app. d, $J=5.1 \mathrm{~Hz}), 1.59$ (2H, br. s); endo: ${ }^{1} \mathrm{H} \mathrm{NMR}\left(500 \mathrm{MHz}, \mathrm{CDCl}_{3}\right.$ ) $\delta 9.62(1 \mathrm{H}, \mathrm{d}, J=1.4 \mathrm{~Hz}, \mathrm{CHO}), 8.13(2 \mathrm{H}, \mathrm{d}, J=8.6 \mathrm{~Hz}), 7.41$ $(2 \mathrm{H}, \mathrm{d}, J=8.6 \mathrm{~Hz}), 6.42(1 \mathrm{H}, \mathrm{dd}, J=.6,3.3 \mathrm{~Hz}), 6.18(1 \mathrm{H}, \mathrm{dd}$, $J=5.6,3.0 \mathrm{~Hz}), 3.41(1 \mathrm{H}, \mathrm{s}), 3.19(1 \mathrm{H}, \mathrm{d}, J=4.9 \mathrm{~Hz}), 3.17$ $(1 \mathrm{H}, \mathrm{s}), 2.96-2.93(1 \mathrm{H}, \mathrm{m}), 1.77-1.66(2 \mathrm{H}, \mathrm{m})$; endo/exo mixture: ${ }^{13} \mathrm{C}$ NMR $\left(126 \mathrm{MHz}, \mathrm{CDCl}_{3}\right) \delta$ 202.2, 201.6, 151.7, $150.7,146.6,146.5,139.1,137.1,136.1,134.1,128.8,128.3$, $123.9,123.4,61.2,59.6,48.5,48.0,47.7,47.2,45.7,45.6,45.2$, 45.1; due to fragmentation, mass spectrometric analysis of the parent aldehydes was not possible; $\mathrm{m} / \mathrm{z}$ of corresponding alcohol (CI): $246.1\left(\mathrm{M}+\mathrm{H}^{+}\right)$.

endo-3-Propylbicyclo[2.2.1]hept-5-ene-2-carbaldehyde and exo- 3-propylbicyclo[2.2.1] hept-5-ene-2-carbaldehyde Table 3 Entry 5. Secondary amine salt $(0.1 \mathrm{mmol}, 1 \mathrm{~mol} \%)$ was dissolved in methanol $(9.5 \mathrm{~mL})$ and water $(0.5 \mathrm{~mL})$. The mixture was stirred at $25{ }^{\circ} \mathrm{C}$. trans-2-Hexenal $(1.16 \mathrm{~mL}, 10$ mmol) was added and the mixture stirred for 10 minutes before freshly distilled cyclopentadiene $(2.00 \mathrm{~mL}, 25 \mathrm{mmol})$ was added. After $18 \mathrm{~h}$ the solvent was removed under reduced pressure $\left(100 \mathrm{mbar}, 25^{\circ} \mathrm{C}\right)$. Water $(20 \mathrm{~mL})$ and diethyl ether $(20 \mathrm{~mL})$ were added to the residue and the aqueous layer extracted with diethyl ether $(2 \times 20 \mathrm{~mL})$. The organics were dried over sodium sulfate and the solvent removed under reduced pressure $\left(100 \mathrm{mbar}, 25{ }^{\circ} \mathrm{C}\right)$. Chloroform $(4 \mathrm{~mL})$ was added to the residue followed by a 1:1 water and TFA mixture ( $4 \mathrm{~mL})$. The biphase was vigorously stirred for 2 hours. The reaction was then quenched with saturated sodium carbonate solution and the aqueous layer extracted with diethyl ether $(3 \times$ $20 \mathrm{~mL}$ ). The combined organics were dried over sodium sulfate and the solvent removed under reduced pressure (100 mbar, 25 $\left.{ }^{\circ} \mathrm{C}\right)$. The product was isolated by flash chromatography $(5 \%$ diethyl ether in petroleum ether) to give a colourless oil. $v_{\max }$ (ATR)/cm ${ }^{-1}$ : 2957, 2918, 2870, 1717; exo ${ }^{1} \mathrm{H}$ NMR (400 MHz, $\left.\mathrm{CDCl}_{3}\right) \delta 9.77(1 \mathrm{H}, \mathrm{d}, J=2.7 \mathrm{~Hz}, C H \mathrm{O}, 6.20(1 \mathrm{H}, \mathrm{dd}, J=5.6$, $3.1 \mathrm{~Hz}), 6.13(1 \mathrm{H}, \mathrm{dd}, J=5.6,2.9 \mathrm{~Hz}), 3.01(1 \mathrm{H}, \mathrm{s}, J=1.3 \mathrm{~Hz})$, $2.87(1 \mathrm{H}, \mathrm{s}), 2.32-2.24(1 \mathrm{H}, \mathrm{m}), 1.77-1.73(1 \mathrm{H}, \mathrm{m}), 1.55-1.04$ $(6 \mathrm{H}, \mathrm{m}), 0.90(3 \mathrm{H}, \mathrm{t}, J=7.1 \mathrm{~Hz})$; endo ${ }^{1} \mathrm{H}$ NMR $(400 \mathrm{MHz}$, $\left.\mathrm{CDCl}_{3}\right) \delta 9.36(1 \mathrm{H}, \mathrm{d}, J=3.4 \mathrm{~Hz}), 6.27(1 \mathrm{H}, \mathrm{dd}, J=5.7,3.2$ $\mathrm{Hz}), 6.05(1 \mathrm{H}, \mathrm{dd}, J=5.7,2.8 \mathrm{~Hz}), 3.11(1 \mathrm{H}, \mathrm{s}), 2.66(1 \mathrm{H}, \mathrm{d}, J$ $=1.5 \mathrm{~Hz}), 2.37(1 \mathrm{H}, \mathrm{dd}, J=7.8,3.4 \mathrm{~Hz}), 1.71-1.65(1 \mathrm{H}, \mathrm{m})$, $1.56-1.03(6 \mathrm{H}, \mathrm{m}), 0.90(3 \mathrm{H}, \mathrm{t}, J=7.1 \mathrm{~Hz})$. endo and exo mixture: ${ }^{13} \mathrm{C}$ NMR $\left(101 \mathrm{MHz}, \mathrm{CDCl}_{3}\right) \delta 205.2,204.1,138.9$, 136.2, 136.2, 132.9, 60.2, 58.9, 47.4, 47.2, 46.6, 45.2, 42.1, 38.2, 36.6, 21.8, 21.7, 14.3; m/z (CI): $181\left(\mathrm{M}+{ }^{+} \mathrm{CH}_{5}\right), 163$ $\left(\mathrm{M}-\mathrm{H}^{-}\right)$. 
endo-3-Isopropylbicyclo[2.2.1] hept-5-ene-2-carbaldehyde and exo-3-Isopropylbicyclo[2.2.1] hept-5-ene-2carbaldehyde Table 3 Entry 6. Secondary amine salt $(0.1$ mmol, $1 \mathrm{~mol} \%)$ was dissolved in methanol $(9.5 \mathrm{~mL})$ and water $(0.5 \mathrm{~mL})$. The mixture was stirred at $25^{\circ} \mathrm{C}$. trans-4-Methyl-2hexenal $(1.16 \mathrm{~mL}, 10 \mathrm{mmol})$ was added and the mixture stirred for 10 minutes before freshly distilled cyclopentadiene (2.00 $\mathrm{mL}, 25 \mathrm{mmol}$ ) was added. After $18 \mathrm{~h}$ the solvent was removed under reduced pressure $\left(100 \mathrm{mbar}, 25{ }^{\circ} \mathrm{C}\right)$. Water $(20 \mathrm{~mL})$ and diethyl ether $(20 \mathrm{~mL})$ were added to the residue and the aqueous layer extracted with diethyl ether $(2 \times 20 \mathrm{~mL})$. The organics were dried over sodium sulfate and the solvent removed under reduced pressure (100 mbar, $\left.25{ }^{\circ} \mathrm{C}\right)$. Chloroform $(4 \mathrm{~mL})$ was added to the residue followed by a $1: 1$ water and TFA mixture $(4 \mathrm{~mL})$. The biphase was vigorously stirred for 2 hours. The reaction was then quenched with saturated sodium carbonate solution and the aqueous layer extracted with diethyl ether $(3 \times 20 \mathrm{~mL})$. The combined organics were dried over sodium sulfate and the solvent removed under reduced pressure $\left(100 \mathrm{mbar}, 25^{\circ} \mathrm{C}\right)$. The product was isolated by flash chromatography (5\% diethyl ether in petroleum ether) to give a colourless oil. $v_{\max }(\mathrm{ATR}) / \mathrm{cm}^{-}$ ${ }^{1}: 2957,2911$, 2895, 2870, 1701; exo ${ }^{1} \mathrm{H}$ NMR (400 MHz, $\left.\mathrm{CDCl}_{3}\right) \delta 9.78(1 \mathrm{H}, \mathrm{d}, J=2.6 \mathrm{~Hz}, \mathrm{CHO}), 6.19(1 \mathrm{H}, \mathrm{dd}, J=5.6$, $3.1 \mathrm{~Hz}), 6.15(1 \mathrm{H}, \mathrm{dd}, J=5.6,2.8 \mathrm{~Hz}), 3.04-3.00(1 \mathrm{H}, \mathrm{m}), 2.96$ $(1 \mathrm{H}, \mathrm{s}), 1.92-1.84(2 \mathrm{H}, \mathrm{m}), 1.51-1.40(2 \mathrm{H}, \mathrm{m}), 1.08-0.97(1 \mathrm{H}$, $\mathrm{m}), 0.94(3 \mathrm{H}, \mathrm{d}, J=6.2 \mathrm{~Hz}), 0.84(3 \mathrm{H}, \mathrm{d}, J=6.4 \mathrm{~Hz})$; endo ${ }^{1} \mathrm{H}$ NMR (400 MHz, CDCl 3 ) $\delta 9.36(1 \mathrm{H}, \mathrm{d}, J=3.4 \mathrm{~Hz}, \mathrm{CHO}), 6.26$ $(1 \mathrm{H}, \mathrm{dd}, J=5.7,3.3 \mathrm{~Hz}), 6.06(1 \mathrm{H}, \mathrm{dd}, J=5.7,2.8 \mathrm{~Hz}), 3.11$ $(1 \mathrm{H}, \mathrm{s}, \mathrm{CH}), 2.87-2.83(1 \mathrm{H}, \mathrm{m}), 2.51-2.47(1 \mathrm{H}, \mathrm{m}), 1.51-1.39$ $(1 \mathrm{H}, \mathrm{m}), 1.34-1.29(1 \mathrm{H}, \mathrm{m}), 1.01(1 \mathrm{H}, \mathrm{d}, J=6.5 \mathrm{~Hz}), 0.91(1 \mathrm{H}$, $\mathrm{d}, J=6.6 \mathrm{~Hz})$; endolexo mixture ${ }^{13} \mathrm{C} \mathrm{NMR}\left(101 \mathrm{MHz}, \mathrm{CDCl}_{3}\right)$ $\delta$ 205.4, 204.3, 139.1, 136.4, 135.9, 133.2, 58.8, 58.1, 50.4, 50.2, 47.0, 46.6, 45.3, 45.3, 45.1, 45.1, 32.9, 32.6, 22.1, 22.1, 21.9, 21.6; $\mathrm{m} / \mathrm{z}(\mathrm{CI}): 181\left(\mathrm{M}+{ }^{+} \mathrm{CH}_{5}\right), 163\left(\mathrm{M}-\mathrm{H}^{-}\right)$.

\section{General procedure for monitoring reaction between cinnamaldehyde and $N$-methyl pyrrole}

\section{(S)-3-(1-Methyl-1H-pyrrol-2-yl)-3-phenylpropan-1-ol.}

Secondary amine salt $(10 \mathrm{~mol} \%, 0.21 \mathrm{mmol})$ was dissolved in THF (4 mL) and water $(0.6 \mathrm{~mL})$. The mixture was stirred at 25 ${ }^{\circ} \mathrm{C}$ and after 5 minutes cinnamaldehyde $(250 \mu \mathrm{L}, 2 \mathrm{mmol})$ was added. Stirring was continued for 10 minutes before $N$-methyl pyrrole $(530 \mu \mathrm{L}, 5.97 \mathrm{mmol})$ was added in one portion. Aliquats $(100 \mu \mathrm{L})$ were periodically taken and added to a mixture of sodium borohydride $(5 \mathrm{mg}, 0.13 \mathrm{mmol})$ in ethanol (1 $\mathrm{mL})$. After 15 minutes the reduction was quenched with saturated sodium bicarbonate solution $(5 \mathrm{~mL})$ and extracted with dichloromethane $(2 \times 10 \mathrm{~mL})$. The organics were dried over sodium sulfate and the solvent removed under reduced pressure. The residue was analysed by ${ }^{1} \mathrm{H}$ NMR to determine reaction conversion using resonances: $4.33 \mathrm{ppm}(2 \mathrm{H}$, dd, $\left.\mathrm{CH}_{2} \mathrm{OH}\right)$ from cinnamyl alcohol and $4.15(1 \mathrm{H}, \mathrm{t}, \mathrm{PhCH})$ from the product. The product was isolated using flash chromatography (16\% ethyl acetate in petroleum ether) as a colourless oil. $v_{\max }(\mathrm{ATR}) / \mathrm{cm}^{-1}$ 3246, 2965, 2930, 2864; ${ }^{1} \mathrm{H}$ NMR (500 MHz, $\left.\mathrm{CDCl}_{3}\right) \delta$ 7.34-7.28 $(2 \mathrm{H}, \mathrm{m}, \mathrm{CH}), 7.24-7.17$ $(3 \mathrm{H}, \mathrm{m}, \mathrm{CH}), 6.58-6.54(1 \mathrm{H}, \mathrm{m}, \mathrm{CH}), 6.20-6.14(2 \mathrm{H}, \mathrm{m}, \mathrm{CH})$, $4.15(1 \mathrm{H}$, app. t, $J=7.6 \mathrm{~Hz}, \mathrm{PhCH}), 3.74-3.57(2 \mathrm{H}, \mathrm{m}$, $\left.\mathrm{CH}_{2} \mathrm{OH}\right), 3.32\left(3 \mathrm{H}, \mathrm{s}, \mathrm{NCH}_{3}\right), 2.42-2.31(1 \mathrm{H}, \mathrm{m}, \mathrm{CH} H), 2.17-$ $2.07(1 \mathrm{H}, \mathrm{m}, \mathrm{CHH}), 1.78(1 \mathrm{H}, \mathrm{s}, \mathrm{OH}) ;{ }^{13} \mathrm{C}$ NMR $(126 \mathrm{MHz}$, $\left.\mathrm{CDCl}_{3}\right) \delta 143.6,135.0,128.6,128.0,126.4,121.9,106.4$, $105.8,60.6,39.5,39.0,33.9 ; \mathrm{m} / \mathrm{z}(\mathrm{ES}): 216.0\left(\mathrm{M}+\mathrm{H}^{+}\right)$.

\section{Acknowledgements}

The authors thank EPSRC for financial support and the National Mass Spectrometry Facility, Swansea, U.K., for highresolution spectra..

\section{Notes and references}

${ }^{a}$ School of Chemistry, Main Building, Cardiff University, Park Place, Cardiff, CF10 3AT, U.K.

${ }^{b}$ WestCHEM, Department of Pure and Applied Chemistry, University of Strathclyde, Glasgow, G1 1XL, U.K.

$\dagger$ Supplementary crystallographic data (CCDC 1017471) can be obtained free of charge from The Cambridge Crystallographic Data Centre via www.ccdc.cam.ac.uk/data_request/cif.

Electronic Supplementary Information (ESI) available: ${ }^{1} \mathrm{H}$ and ${ }^{13} \mathrm{C}$ spectra for compounds reported.

1 K. N. Houk and P. H.-Y. Cheong, Nature, 2008, 455, 309.

2 (a) P. H.-Y. Cheong, C. Y. Legault, J. M. Um, N. ÇelebiÖlçüm and K. N. Houk, Chem. Rev., 2011, 111, 5042; (b) H. Yang and M. W. Wong, J. Org. Chem., 2011, 76, 7399; (c) Y. Wang, J. Wang, J. Su, F. Huang, L. Jiao, Y. Liang, D. Yang, S. Zhang, P. A. Wender and Z.-X. Yu, J. Am. Chem. Soc., 2007, 129, 10060; (d) S. T. Schneebeli, M. L. Hall, R. Breslow and R. Friesner, J. Am. Chem. Soc., 2009, 131, 3965; (e) J. M. Brown and R. J. Deeth, Angew. Chem., Int. Ed., 2009, 48, 4476.

3 (a) D. W. C. MacMillan, Nature, 2008, 455, 304. (b) Asymmetric Organocatalysis Workbench Edition. 2 Volumes, Science of Synthesis, B. List and K. Maruoka Eds.; Thieme: Stuttgart, 2012.

4 J. B. Brazier and N. C. O. Tomkinson, Top. Curr. Chem. 2010, 291, 281.

5 A. Erkkilae, I. Majander and P. M. Pihko, Chem. Rev., 2007, 107, 5416.

6 F. Giacalone, M. Gruttadauria, P. Agrigento and R. Noto, Chem. Soc. Rev., 2012, 41, 2406.

7 K. A. Ahrendt, C. J. Borths, and D. W. C. MacMillan, J. Am. Chem. Soc., 2000, 122, 4243.

8 (a) G. Evans, T. J. K. Gibbs, R. L. Jenkins, S. J. Coles, M. B. Hursthouse, J. A. Platts, and N. C. O. Tomkinson, Angew. Chem. Int Ed., 2008, 47, 2820; (b) J. B. Brazier, G. Evans, T. J. K. Gibbs, S. J. Coles, M. B. Hursthouse, J. A. Platts, and N. C. O. Tomkinson, Org. Lett., 2009, 11, 133.

9 (a) D. Seebach, R. Gilmour, U. Grošelj, G. Deniau, C. Sparr, M.-O. Ebert, A. K. Beck, L. B. McCusker, D. Šišak, and T. Uchimaru, Helv. Chim. Acta, 2010, 93, 603; (b) D. Seebach, U. Grošelj, W. B. Schweizer, S. Grimme, and C. Muck-Lichtenfeld, Helv. Chim. Acta, 2010, 93, 1; (c) U. Grošelj, W. B. Schweizer, M.-O. Ebert, and D. Seebach, Helv. Chim. Acta, 2009, 92, 1; (d) D. Seebach, U. Grošelj, D. M. Badine, W. B. Schweizer, and A. K. Beck, Helv. Chim. Acta, 2008, 91, 1999.

10 J. H. Rowley and N. C. O. Tomkinson, In Asymmetric Synthesis II; M. Christmann and S. Bräse, Eds.; Wiley-VCH: Weinheim, 2012; pp 29-34.

11 J. B. Brazier, K. M. Jones, J. A. Platts and N. C. O. Tomkinson, Angew. Chem. Int. Ed., 2011, 50, 1613.

12 W. P. Jencks, Catalysis in Chemistry and Enzymology; McGraw Hill Text: New York, 1969.

13 For a review on the use of imidazolidinone catalysts see: G. Lelais, and D. W. C. MacMillan, Aldrichimica Acta 2006, 39, 79.

14 For a review on the use of diarylprolinol ether catalysts see: A. Mielgo, and P. Claudio, Chem. Asian J. 2008, 3, 922.

15 J. B. Brazier, G. P. Hopkins, M. Jirari, S. Mutter, R. Pommereuil, L. Samulis, J. A. Platts and N. C. O. Tomkinson, Tetrahedron Lett., 2011, $\mathbf{5 2}, 2783$.

16 (a) J. L. Cavill, J.-E. Peters, and N. C. O. Tomkinson, Chem. Commun., 2003, 728; (b) J. L. Cavill, R. L. Elliott, G. Evans, I. L. Jones, J. A. Platts, A. M. Ruda, and N. C. O. Tomkinson, Tetrahedron, 2006, 62 , 410; (c) J. B. Brazier, J. L. Cavill, R. L. Elliott, G. Evans, T. J. K. Gibbs, I. L. Jones, J. A. Platts, and N. C. O. Tomkinson, Tetrahedron, 2009, 65, 9961.

17 For studies on alternative secondary amine scaffolds see: (a) E. Gould, T. Lebl, A. M. Z. Slawin, M. Reid, T. Davies, and A. D. Smith, Org. 
Biomol. Chem. 2013, 11, 7877; (b) I. Suzuki, M. Ando, R. Shimabara, A. Hirata and K. Takeda, Org. Biomol. Chem., 2011, 9, 3033; (c) Q. Li, W.Y. Wong, W.-H. Chan and A. W. M. Lee, Adv. Synth. Catal., 2010, 352, 2142-2146; (d) Y. Langlois, A. Petit, P. Rémy, M.-C. Scherrmann and C. Kouklovsky, Tetrahedron Lett., 2008, 49, 5576; (e) H. He, B. J. Pei, H.-H. Chou, T. Tian, W.-H. Chan and A. W. M. Lee, Org. Lett., 2008, 10, 2421. (f) M. Lemay, L. Aumand and W. W. Ogilvie, Adv. Synth. Catal., 2007, 349, 441; (g) M. Lemay and W. W. Ogilvie, Org. Lett., 2005, 7, 4141

18 G. J. S. Evans, K. White, J. A. Platts and N. C. O. Tomkinson, Org. Biomol. Chem., 2006, 4, 2616.

19 L. Samulis and N. C. O. Tomkinson, Tetrahedron, 2011, 67, 4263.

20 A. Hall, L. D. Harris, C. L. Jones, and N. C. O. Tomkinson, Tetrahedron Lett. 2003, 44, 111.

21 For a review on dienamines in catalysis, see: D. B. Ramachary and Y. V. Reddy, Eur. J. Org. Chem., 2012, 865.

22 endo:exo ratios observed for transformations accelerated with imidazolidinones have been rationalised through DFT calculations: (a) C. Alleman, R. Gordillo, F. R. Clemente, P. H.-Y Cheong and K. N. Houk, Acc. Chem. Res. 2004, 37, 558; (b) R. Gordillo and K. N. Houk, J. Am. Chem. Soc. 2006, 128, 3543.

23 J. F. Austin and D. W. C. MacMillan, J. Am. Chem. Soc. 2002, 124, 1172.

24 S. Lakhdar and H. Mayr, Chem. Commun., 2011, 47, 1866. 